\title{
Assessments of HLA-I Specificities of Anti-HLA-I Monoclonal Antibodies Using Solid Phase Bead Arrays
}

Anita J. Zaitouna ${ }^{1}$, Daniel S. Ramon ${ }^{2}$ and Malini Raghavan ${ }^{1, *}$

\author{
1Department of Microbiology and Immunology, Michigan Medicine, $1150 \mathrm{~W}$. Medical Center Drive, \\ University of Michigan, Ann Arbor, MI 48109, USA; '2Department of Laboratory Medicine and Pathology, \\ Mayo Clinic, Arizona, USA \\ *For correspondence: malinir@umich.edu
}

[Abstract] Human leukocyte antigen class I (HLA-I) molecules are a group of structurally-related cell surface proteins with a high degree of variability within the population. While only up to six variants are expressed in an individual person, the whole population contains thousands of different variants. The ability to distinguish specific variants is important in the clinic to determine compatibility during organ and bone marrow transplantation and in the laboratory to study the biological properties of individual variants. Solid phase bead arrays contain purified, individually identifiable HLA-I molecules that can be used to determine antibody specificity for individual HLA-I proteins. This method is high-throughput, highly specific, and allows for simultaneous screening of antibodies against multiple HLA-I allotypes. The beads are particularly useful for screening patient sera for the presence of donor-specific antibodies against individual HLA-I variants (which can arise during pregnancy, blood transfusion, or organ transplantation). Alternate approaches, such as the use of individual HLA-I-expressing cell lines, are more time consuming, and such cell lines are difficult to procure and standardize. The HLA-I beads are also useful to study HLA-I specificity and selectivity for other receptors and binding partners.

Keywords: Luminex beads, Immunology, Specificity, Affinity, Monoclonal antibodies, Bead array

[Background] Human leukocyte antigen molecules are classified as class I (HLA-I) or class II (HLA-II). Generally, HLA-I are found on all nucleated cells and bind to T cell receptors of $C D 8^{+} T$ cells, whereas HLA-II are found on specialized immune cells called antigen presenting cells and bind to $T$ cell receptors of $\mathrm{CD}^{+} \mathrm{T}$ cells. The major receptors for HLA-I and HLA-II proteins are T cell receptors in conjunction with the co-receptors CD8 or CD4, although other receptors, proteins, and antibodies are known to functionally interact with both sets of molecules. HLA-I molecules comprise a highly polymorphic heavy chain, an invariant light chain ( $\beta 2$-microglbulin), and a short peptide. Solid phase bead arrays containing HLA molecules are widely used to screen patient sera for monitoring the presence of antibodies against specific HLA-I and HLA-II allotypes (Lachmann et al., 2013), as such antibodies mediate transplant rejection in organ and bone marrow transplantations. Solid phase bead arrays containing individual HLA-I (or HLA-II) proteins can also be used to identify HLA interactions with various receptors and assembly factors (for example, Garcia-Beltran et al., 2016 and llca et al., 2019). In Yarzabek et al. (2018), monoclonal antibodies against known epitopes on HLA-I heavy chains, called Bw4 or Bw6 epitopes (Müller et al., 1989; residues 77-83 of the HLA-I heavy chain) were bound to purified HLA-I-containing 
solid phase beads, with the goal of determining each antibody's selectivity for Bw4 or Bw6 epitopes on individual HLA-I molecules. W6/32, a pan HLA-I antibody (Barnstable et al., 1978; Raghavan et al., 2019), was used to account for potential variations in HLA-I protein levels on the beads. Here we describe the detailed procedures for using HLA-I bead arrays to measure anti-Bw4 and anti-Bw6 selectivity and specificity for various HLA-I molecules, expanding on the protocols used in Yarzabek et al. (2018).

\section{Materials and Reagents}

1. $1.5 \mathrm{ml}$ centrifuge tubes (Thermo Fisher Scientific, catalog number: 05-408-129)

2. $30 \mathrm{~K} \mathrm{MWCO}$ centricon filters (MilliporeSigma, catalog number: UFC903008)

3. Biotinylated anti-Bw6 (Thermo Fisher Scientific, One Lambda Inc., catalog number: BiH0038, store at $4{ }^{\circ} \mathrm{C}$ )

4. Biotinylated anti-Bw4 (Thermo Fisher Scientific, One Lambda Inc., catalog number: BiH0007, store at $\left.4{ }^{\circ} \mathrm{C}\right)$

5. W6/32 purified from supernatants or ascites derived from hybridoma line ATCC HB-95 using a protein G column (GE Lifesciences, catalog number: 17040401 , store at $4{ }^{\circ} \mathrm{C}$ in $20 \%$ ethanol) (Springer, 2001) or procured as purified protein from a commercial source (for example, Bio $X$ Cell, catalog number: BE0079)

6. EZ-Link NHS-PEG 4 -Biotin (Thermo Fisher Scientific, catalog number: 21330 , store at $-20{ }^{\circ} \mathrm{C}$ )

7. Labscreen Single Antigen HLA class-I Combination bead suspension array, which contains 97 common HLA-A, - $B$, and $-C$ bound beads derived from individual cell lines, a positive control bead sample, and a negative control bead sample, where each bead is distinguished based on the fluorescence intensity readout (Thermo Fisher Scientific, One Lambda Inc., Labscreen ${ }^{\circledR}$, catalog number: Class-I-LS1A04NC, store at $4{ }^{\circ} \mathrm{C}$ )

Note: For flow cytometry-based experiments, FlowPRA Single Antigen Beads, which contain 32 total beads (8 HLA-I and one negative control bead sample in 4 groups, where the 9 beads are labeled with differing amounts of fluorophore that can be measured in the PE channel of a flow cytometer) can be used and supplemented with additional kits if necessary (Thermo Fisher Scientific, One Lambda Inc., catalog number: FL1HD, store at $4{ }^{\circ} \mathrm{C}$ )

8. 10x LabScreen Wash buffer (Thermo Fisher Scientific, One Lambda Inc., Labscreen ${ }^{\circledR}$, catalog number: LSPWABUF, store at $-65^{\circ} \mathrm{C}$ or lower until open, then store at $4{ }^{\circ} \mathrm{C}$ for up to three months)

9. PE Conjugated Streptavidin (Thermo Fisher Scientific Inc., One Lambda Inc., Labscreen ${ }^{\circledR}$, catalog number: LT-SAPE, store at $4{ }^{\circ} \mathrm{C}$ )

10. FITC Conjugated Streptavidin (Biolegend, catalog number: 405201 , store at $4{ }^{\circ} \mathrm{C}$ )

11. 1x PBS buffer (Life Technologies Corporation, Gibco, catalog number: 10010-023) 


\section{Equipment}

1. 0.2 to $2 \mu$ pipette (Thermo Fisher Scientific, catalog number: FBE00002)

2. 2 to $20 \mu$ pipette (Thermo Fisher Scientific, catalog number: FBE00020)

3. 20 to $200 \mu$ pipette (Thermo Fisher Scientific, catalog number: FBE00200)

4. 10 to $1,000 \mu \mathrm{l}$ pipette (Thermo Fisher Scientific, catalog number: FBE01000)

5. Eppendorf Centrifuge 5425 (VWR, catalog number: 5405000441)

6. Eppendorf Centrifuge 5810R (VWR, catalog number: 022628187)

7. LabScan 100 flow analyzer (Luminex Corp., Luminex Analyzer, Luminex 100/200 System) or flow cytometer such as BD LSRFortessa (Becton, Dickinson and Company, Flow cytometer, LSRFortessa)

\section{Software}

1. HLA Fusion Software (Thermo Fisher Scientific Inc., One Lambda Inc., https://www.onelambda.com/en/product/hla-fusion.html) or FlowJo (Becton, Dickinson and Company, Flowjo, https://www.flowjo.com)

\section{Procedure}

A. Biotinylation of $W 6 / 32$

1. Buffer exchange (via dialysis in a centricon filter) of W6/32 into PBS Buffer.

Note: W6/32 can be purchased commercially in a purified form or can be purified from ascites fluid or cell supernatant derived with the Hybridoma line (ATCC, HB-95).

2. Immediately before use, add $170 \mu \mathrm{l}$ of ultrapure water to $2 \mathrm{mg}$ of NHS-PEG 4 -Biotin to prepare a $20 \mathrm{mM}$ stock solution.

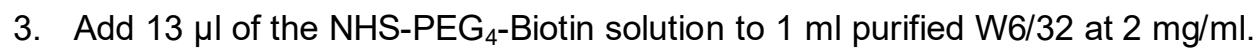

4. Incubate the reaction on ice or at $4{ }^{\circ} \mathrm{C}$ for two hours.

5. Remove non-reacted NHS-PEG - -Biotin by extensive dialysis (6-10 rounds of dilution to maximal volume followed by concentration to minimum specified volume) with a $15 \mathrm{ml} 30 \mathrm{~K} \mathrm{MWCO}$ centricon filter.

6. Store antibody at $4{ }^{\circ} \mathrm{C}$.

B. Reagent set-up

1. Dilute $10 x$ wash buffer to $1 x$ with distilled water.

2. Gently mix Labscreen Single Antigen HLA class-I Combination bead array suspension.

C. Sample preparation

Note: Follow procedure as described by OneLambda packet insert. 
1. Mix $20 \mu \mathrm{l}$ of biotinylated anti-Bw4, biotinylated anti-Bw6, or biotinylated $W 6 / 32$ at multiple dilutions ranging from $1 / 50$ to $1 / 200$ of the stock concentration of purified biotinylated antibodies that were prepared in-house (W6/32; $2 \mathrm{mg} / \mathrm{ml})$ or through a commercial source (anti-Bw4 and anti-Bw6) with $5 \mu$ L Labscreen Single Antigen HLA class-I Combination bead array suspension.

2. Incubate for $30 \mathrm{~min}$ at room temperature.

3. Add $1 \mathrm{ml}$ of $1 \mathrm{x}$ wash buffer, mix gently.

4. Centrifuge tube at $9,300 \times g$ for 2 min.

5. Discard supernatant by aspirating sample.

6. Repeat Steps C3-C5 two more times.

7. Resuspend beads with 1:100 streptavidin-PE directly from the tube (made with $1 \mathrm{x}$ wash buffer).

8. Incubate for $30 \mathrm{~min}$ at room temperature.

9. Repeat Steps C3-C5 twice.

10. Resuspend beads with $80 \mu \mathrm{l} 1 \mathrm{x}$ wash buffer (proceed to Procedure $\mathrm{D}$ or store at $4{ }^{\circ} \mathrm{C}$ for up to $24 \mathrm{~h})$.

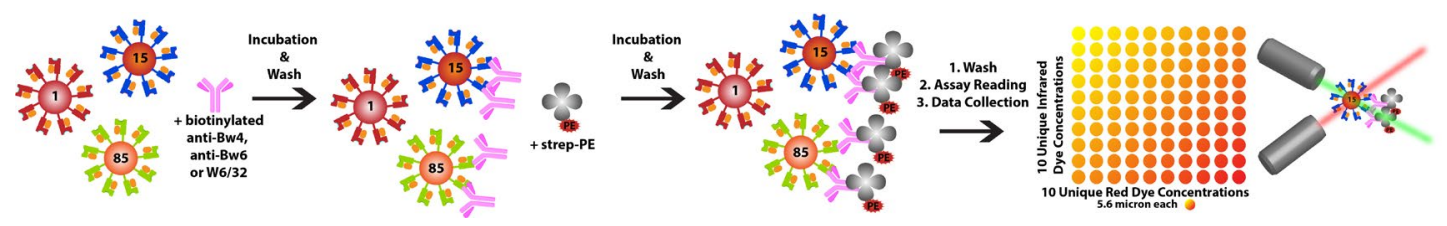

Figure 1. Labscreen Single Antigen Immunoaffinity protocol. Labscreen Single Antigen HLA class-I Combination bead (each labeled with identifiable fluorescent dye and HLA-I) suspension array is successively incubated with biotinylated antibody, washed, incubated with streptavidin-PE, washed, and finally measured on the Labscreen flow analyzer.

D. Data acquisition on LabScan

Note: Follow procedure as described by OneLambda packet insert.

1. Requires an acquisition template, downloadable from OneLambda website https://www.onelambda.com/en/product/labscan.html.

2. Create a file name for the samples to be run.

3. Confirm template settings.

Sample Volume

Sample Time-Out

Double discriminator gate Low Limit

Double discriminator gate High Limit

Number and ID of beads

Minimum events collected

4. Enter sample IDs.

5. Load plate onto platform.

6. Click start button to initiate.
$50 \mu \mathrm{l}$

$80 \mathrm{~s}$

8,000

16,000

100 events per bead 
7. Save data output in a .csv file and export.

8. Open Fusion OL software.

9. Select the appropriate output .csv file.

10. Select the appropriate Catalog ID file.

11. Select the negative control.

12. Click Import, then analyze each sample.

E. Alternative Method: Flow Cytometry

The assay can also be performed on a flow cytometer using FlowPRA Single Antigen Beads instead of Labscreen Single Antigen HLA class-I Combination bead array suspensions.

1. Reagent set-up

a. Dilute $10 \mathrm{x}$ wash buffer to $1 \mathrm{x}$ with distilled water.

b. Gently mix FlowPRA Single Antigen Beads.

2. Sample preparation (follow procedure as described by OneLambda packet insert)

a. Mix $20 \mu \mathrm{l}$ of biotinylated anti-Bw4, biotinylated anti-Bw6, or biotinylated W6/32 at multiple dilutions (undiluted, 1:25, 1:50, and 1:100) with $5 \mu$ FlowPRA Single Antigen Beads for each group and antibody respectively.

Note: Fluorescently labeled antibodies can be used in place of biotinylated antibodies.

b. Incubate for $30 \mathrm{~min}$ at room temperature.

c. Add $1 \mathrm{ml}$ of $1 \mathrm{x}$ wash buffer, mix gently.

d. Centrifuge tube at $9,000 \times g$ for 2 min.

e. Discard supernatant by aspirating sample.

f. Repeat Steps E2c to E2e two more times.

g. Resuspend beads with 1:200 streptavidin-FITC (made with 1x wash buffer).

h. Incubate for $30 \mathrm{~min}$ at room temperature.

i. Repeat Steps E2c to E2e twice.

j. Resuspend beads with $100 \mu \mathrm{l} 1 \mathrm{x}$ wash buffer (proceed to Step E3 or store at $4{ }^{\circ} \mathrm{C}$ for up to $24 \mathrm{~h}$ after fixing samples).

3. Acquire measurements for each group using a flow cytometer with at least two channels, a PE channel and a FITC channel.

Note: The method of acquisition depends on the flow cytometer used.

4. Gate on each bead population (known PE channel fluorescence intensity for each HLA-I-bead in the group as described in packet insert).

5. Measure histogram of streptavidin-FITC geometric mean fluorescence intensity.

\section{Data analysis}

Follow procedure as described by OneLambda packet insert for the Luminex LabScan. Briefly, data analysis is performed similar to flow cytometry analysis, where each pool of HLA-I beads has 
multiple HLA-I-bead conjugates, each of which is uniquely identified based on the fluorescence intensity of the beads (Luminex XMAP technology, Figure 1). Relative binding of the antibodies (PE signal) are assessed by first correcting for non-specific antibody binding to a negative control bead (which is included as a component of the Labscreen Single Antigen HLA class-I Combination bead suspension array) (example results in Figure 2).

The FlowPRA single antigen bead binding data are collected on a flow cytometer, where the individual HLA-I beads are identified based on different fluorescence intensities in the PE channel (as shown in Figure 3) and the ligand is identified in the FITC channel. Note that a compensation has to be performed for this experiment. Similar to the above protocol, the relative ligand signal (FITC) is corrected with the negative control bead (Figure 3, bead 8, teal), as well as an isotype control. The FlowPRA single antigen beads come as four groups (covering common HLA-A and HLA-B allotypes). To screen a wider array of HLA-I, additional bead groups have to be included.

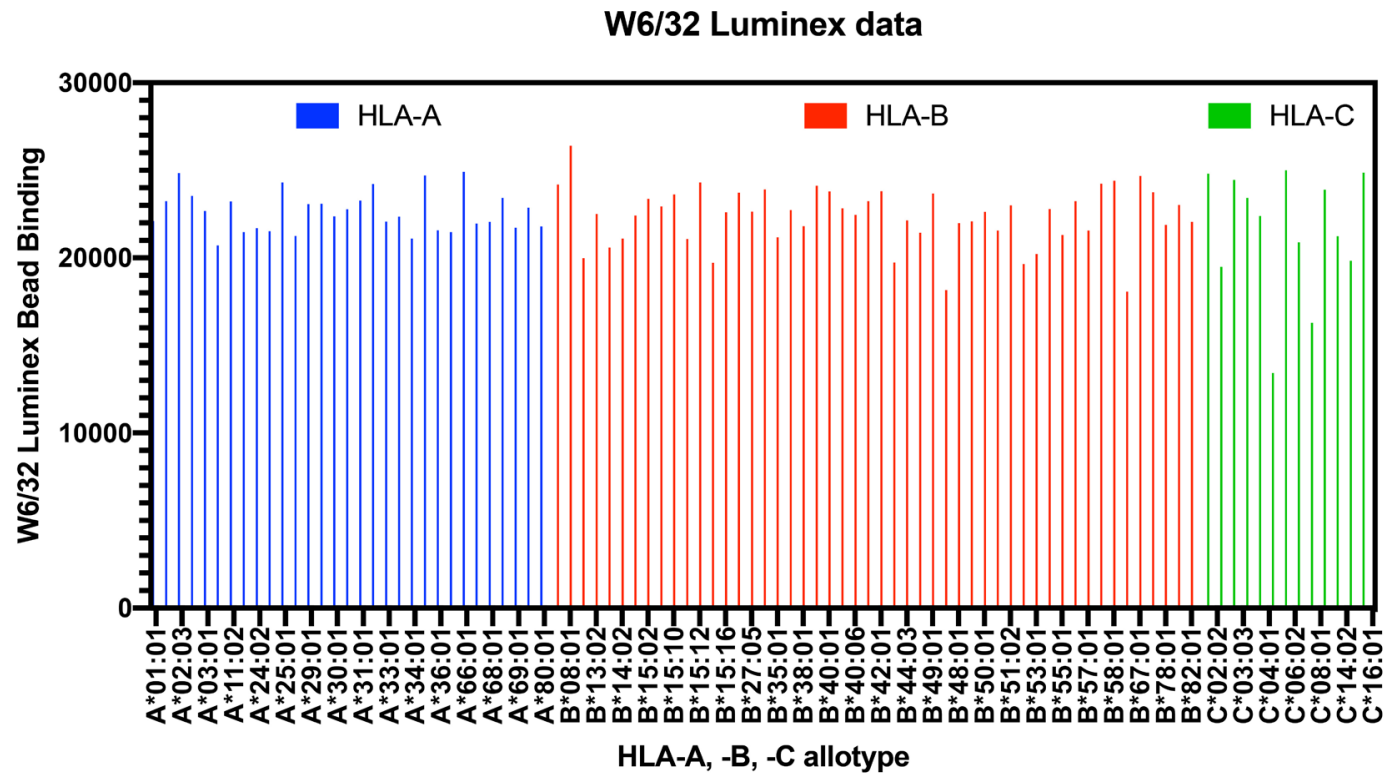

Figure 2. Labscreen Single Antigen Immunoaffinity sample data. Representative data are shown for W6/32 (a pan anti-HLA class-I antibody [Barnstable et al., 1978 and Raghavan et al., 2019]) binding to the indicated HLA-I beads. 

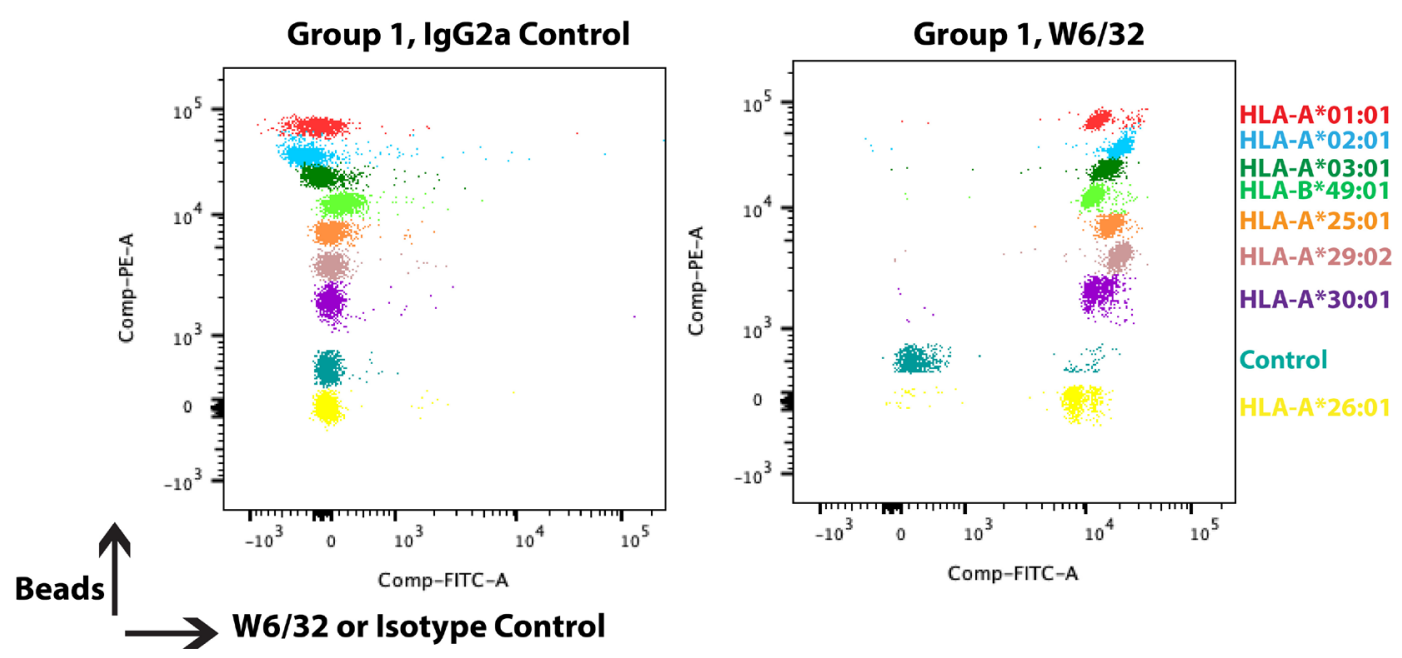

Figure 3. FlowPRA Single Antigen Beads sample data. FlowPRA single antigen beads come as four groups, each containing 8 HLA-I beads and one negative control bead (without HLA-I labeled 8 , and colored teal in dot plots) that are individually identifiable in the PE channel. The beads are successively incubated and washed: biotinylated antibody, buffer, streptavidin-FITC, buffer. They are then measured on a flow cytometer. The dot plots are from a representative experiment, where isotype control, IgG2a (left panel), or W6/32 (right panel) were incubated with Group 1 of FlowPRA single antigen beads.

Additional normalization methods can be performed by taking the background corrected values for anti-Bw4 or anti-Bw6, which bind certain HLA-I molecules (Yarzabek et al., 2018) and calculating the signals as a ratio relative to the W6/32 signal, an antibody known to bind all HLA-I variants (Raghavan et al., 2019) (Figure 2). This radiometric method accounts for potential variations in HLAI levels on the Labscreen Single Antigen HLA class-I Combination bead suspension array.

\section{Acknowledgments}

The authors acknowledge NIH grants (R01 Al044115 (MR), RO1 Al123957 (MR), R21 Al126054 (MR), T32 Al007528 (for support of AZ), T32 Al007413 (for support of AZ)). This protocol was adapted from previous work, see Yarzabek et al. (2018) below.

\section{Competing interests}

The authors do not have any competing interests. 


\section{References}

1. Barnstable, C. J., Bodmer, W. F., Brown, G., Galfre, G., Milstein, C., Williams, A. F. and Ziegler, A. (1978). Production of monoclonal antibodies to group A erythrocytes, HLA and other human cell surface antigens-new tools for genetic analysis. Cell 14(1): 9-20.

2. Garcia-Beltran, W. F., Hölzemer, A., Martrus, G., Chung, A. W., Pacheco, Y., Simoneau, C. R., Rucevic, M., Lamothe-Molina, P. A., Pertel, T., Kim, T. E., Dugan, H., Alter, G., DechanetMerville, J., Jost, S., Carrington, M. and Altfeld, M. (2016). Open conformers of HLA-F are highaffinity ligands of the activating NK-cell receptor KIR3DS1. Nat Immunol 17(9): 1067-1074.

3. Ilca, F. T., Drexhage, L. Z., Brewin, G., Peacock, S. and Boyle, L. H. (2019). Distinct polymorphisms in HLA class I molecules govern their susceptibility to peptide editing by TAPBPR. Cell Rep 29(6): 1621-1632.e1623.

4. Lachmann, N., Todorova, K., Schulze, H., and Schönemann, C. (2013). Luminex ${ }^{\circledR}$ and it's applications for solid organ transplantation, hemoatopoteic stem cell transplantation, and transfusion. Transfus Med Hemother 40(3): 182-189.

5. Müller, C. A., Engler-Blum, G., Gekeler, V., Steiert, I., Weiss, E. and Schmidt, H. (1989). Genetic and serological heterogeneity of the supertypic HLA-B locus specificities Bw4 and Bw6. Immunogenetics 30(3): 200-207.

6. Raghavan, M., Yarzabek, B., Zaitouna, A. J., Krishnakumar, S. and Ramon, D. S. (2019). Strategies for the measurements of expression levels and half-lives of HLA class I allotypes. Hum Immunol 80(4): 221-227.

7. Springer, T. A. (2001). Immunoaffinity chromatography. In: Current Protocols in Protein Science. Chapter 9, Unit 9.5.

8. Yarzabek, B., Zaitouna, A. J., Olson, E., Silva, G. N., Geng, J., Geretz, A., Thomas, R., Krishnakumar, S., Ramon, D. S. and Raghavan, M. (2018). Variations in HLA-B cell surface expression, half-life and extracellular antigen receptivity. Elife 7: 34961. 